\title{
Actualidad de los cuidados paliativos. Revisión de una situación poco estandarizada
}

Publicado en Internet: 10-septiembre-2015

Ángel Segura Huerta: segura_ang@gva.es

Palabras clave:

- Cuidados paliativos

- Pediatría

- Calidad de vida

- Niño

- Adolescente

\section{Salvador Coloma ${ }^{a}$, V. Salvador Coloma ${ }^{b}$, A. Segura Huerta ${ }^{a}, M$. Andrés Morenoc, J. M. Fernández Navarroc ${ }^{c}$ O. M. Niño Gómez ${ }^{\mathrm{a}}$, E. Reche Santos ${ }^{\mathrm{a}}$}

a Servicio de Oncología Médica. Hospital Universitari i Politecnic La Fe. Valencia. España • bescuela de Enfermería. Universidad Católica. Valencia. España • 'Servicio de Oncología Pediátrica.

Hospital Universitari i Politecnic La Fe. Valencia. España.

En los últimos 50 años la medicina ha evolucionado más que en los dos siglos anteriores. Sin embargo, existen en la actualidad multitud de patologías que la medicina contemporánea no ha sido capaz de solventar. Hasta hace tan solo unas décadas, los niños diagnosticados de cáncer fallecían en poco tiempo. Así pues, esto ha ido modificándose y más de la mitad llegan a ser supervivientes a largo plazo. Los cuidados paliativos como especialidad se han desarrollado de forma marcada en los últimos años. Sin embargo, destaca una parcela de ella que todavía se encuentra muy lejos de estar establecida, los cuidados paliativos pediátricos (CPP). Los niños presentan necesidades diferentes que es necesario atender. Se pretende revisar el concepto de CPP, haciendo hincapié en las diferencias que presentan los niños con respecto a los adultos y en su problemática específica.

\section{The current situation of palliative care. Review of a non-standardized situation}

In the last 50 years medicine has evolved more than in the previous two centuries but there are currently many pathologies that modern medicine has not been able to cure. Until a mere few decades ago, children diagnosed with cancer died within a short time. However, this has gradually changed and, nowadays, over half of them become long-term survivors.

Key words:

Palliative care as a medical specialization has markedly developed in recent years. Nevertheless, there is a specific part that is still far from being established, pediatric palliative care (PPC). Children have different needs to be attended. PPC should be reviewed with a special focus on the precise differences present in children and their specific problems.

\section{INTRODUCCIÓN}

A pesar del desarrollo alcanzado en la medicina pediátrica, la muerte en la infancia o adolescencia atribuible a enfermedades no curables todavía es una realidad. En los últimos años se han realizado mejoras sociosanitarias y avances en la medicina que han contribuido a disminuir causas que antiguamente eran mortales. Sin embargo, todavía existen diversas enfermedades incurables, a pesar de los logros alcanzados en la mejoría de la calidad de vida y en la supervivencia.

Los niños presentan características diferentes a los adultos, por tanto la aproximación de los cuidados paliativos (CP) a estos pacientes también debe ser diferente ${ }^{1}$. El principal objetivo es aliviar el 
sufrimiento del niño y su familia mediante un adecuado control de síntomas, apoyo psicológico, social, espiritual y preparación para la muerte y el duelo.

Los cuidados paliativos pediátricos (CPP) nacieron alrededor del paciente con enfermedad oncohematológica en situación terminal. Sin embargo, en la actualidad, dirigirse únicamente a este colectivo sería dedicarse a un pequeño grupo de pacientes perdiendo una amplia serie de niños que podría beneficiarse de los mismos.

En los países desarrollados los niños fallecen por acontecimientos agudos y sus secuelas (por ejempleo, tras un accidente de tráfico), enfermedades derivadas del periodo neonatal y enfermedades crónicas (entre las que destacan el cáncer, las enfermedades neurológicas, neumológicas y cardiológicas). El cáncer es responsable solamente de un pequeño porcentaje de las muertes en edad pediátrica (12-15\%).

El aumento, en las últimas décadas, de la supervivencia en los pacientes oncológicos pediátricos ha hecho que los principales receptores de CPP sean los pacientes con patologías neurológicas. En los datos publicados en 2010 por la Unidad de CPP del Hospital Niño Jesús (Madrid) casi dos de cada tres pacientes que precisan sus servicios no son oncológicos y el grupo mayoritario son los que presentan parálisis cerebral infantil2.

Todo esto pone de manifiesto la importancia de que los profesionales relacionados con la atención pediátrica reciban nociones básicas de CPP, con independencia de que existan especialistas en este campo, hoy en día todavía escasos.

\section{OBJETIVOS Y CARACTERÍSTICAS DIFERENCIALES DE LOS CUIDADOS PALIATIVOS PEDIÁTRICOS}

Los CPP se encargan del cuidado integral del niño y su familia con el objetivo de garantizar su dignidad y el derecho a la autonomía con una atención continuada e individualizada. El acto del médico debe basarse en los cuatro principios fundamentales de la bioética ${ }^{3}$ : beneficencia, no maleficencia, autonomía y justicia. Ello implica que, mientras que el objetivo principal del tratamiento curativo es mejorar la supervivencia del paciente, en el caso del tratamiento paliativo prima la calidad de vida, de donde derivan sus características y objetivos ${ }^{4,5}$ :

- Atención integral del paciente, individualizada y continuada, que considere los aspectos físicos, emocionales, sociales y espirituales.

- Reconocer al paciente no solo de forma individual, sino formando parte de una unidad social y familiar, considerando que el niño y la familia son el conjunto a tratar.

- Las decisiones terapéuticas se tienen que basar en la dignidad del enfermo y la autonomía.

- Crear un ambiente de intimidad, respeto, confort, soporte y comunicación. No solo hay que considerar el lugar físico sino hacer partícipe del mismo a la familia y el equipo sanitario.

- Priorizar la atención en el domicilio, evitando si es posible la hospitalización, tratando de mantener las actividades diarias, adaptadas a las limitaciones del paciente en cada momento. Es en este punto donde las unidades de CPP domiciliarios presentan su mayor utilidad, manteniendo los cuidados del niño sin separarlo de su ambiente.

Las complejidades de los CPP se deben principalmente $a^{6}$ :

- La gran variedad de entidades (neurológicas, cáncer, cardiológicas...) y la evolución, no claramente definida, de alguna de ellas.

- En comparación con los adultos, los pacientes pediátricos candidatos a recibir cuidados paliativos presentan una baja prevalencia y una importante dispersión geográfica, lo que supone un problema en lo que se refiere a la organización y coste asistencial.

- Muchos fármacos se encuentran desarrollados y aprobados para adultos y en multitud de ocasiones no existe información para su uso en la infancia. Lo cual implica que gran parte de los fármacos utilizados en los CPP se prescriben al margen de la ficha técnica. 
- Cabe destacar el papel fundamental de la familia, puesto que las decisiones clínicas, éticas, terapéuticas y sociales (aunque depende de la edad y de estado madurativo del niño) en la mayoría de casos recaen sobre los padres.

- El grado de maduración del niño obliga en muchas ocasiones a modificar el método de comunicación, educación y apoyo.

- El impacto social que produce en el niño y en su familia mantener su papel en la sociedad en el curso de una enfermedad, que puede ser larga e incapacitante.

- El duelo tras la muerte de un niño suele ser más duradero y complicado. A su vez produce una implicación emocional difícil, no solo para la familia, sino también para los profesionales, ya que supone aceptar las limitaciones del tratamiento y la irreversibilidad del cuadro.

- La ausencia de una especialidad médica que se dedique a este fin, puesto que la gran mayoría de los profesionales presentan una falta de formación en lo que se refiere al cuidado de los niños que se encuentran implicados en una enfermedad terminal. Esto se debe a que el número de niños que fallece en comparación con los adultos es bajo, lo cual implica que pocos profesionales, aunque se dediquen al cuidado del niño, se hayan enfrentado a esta situación.

En el Children's Hospital de San Francisco (EE. UU.) se realizó un estudio donde se trataron de encontrar las distintas barreras que dificultan la implantación de los CPP en los diferentes hospitales ${ }^{7}$. Se identificaron unas 26 barreras, siendo las más frecuentes: la incertidumbre sobre el pronóstico (54,6\%), la negación familiar (51\%), la barrera cultural $(47,3 \%)$ y la falta de tiempo por parte de los cuidadores $(47,1 \%)$.

En lo que respecta a la incertidumbre sobre el pronóstico, es importante destacar la amplia variedad de enfermedades (anomalías congénitas, cromosómicas...) para las cuales es muy complicado conocer la evolución y el pronóstico de las mismas. Por tanto, en estos momentos, en los que no queda clara la evolución de la enfermedad, es importante tratar de integrar ambos tipos de tratamiento para asegurar la mejor calidad de vida posible para el niño y sus familiares.

\section{DIFERENTES MODELOS DE CUIDADOS PALIATIVOS}

A medida que ha ido avanzando la sociedad se han ido modificando los modelos de CP. En 1990 la Organización Mundial de la Salud (OMS) definía los CP como el "cuidado integral" de los pacientes que no habían respondido a tratamiento con intención curativa $^{8}$. Las principales dificultades que conlleva este modelo son que se trata de un modelo rígido, excluyente, donde para iniciar los CP es necesario terminar el tratamiento curativo, sin posibilidad de interacción entre ambos y relegando la paliación a un estado de terminalidad (Fig. 1).

En 1993 el Children's Hospice Internacional introdujo un nuevo modelo como alternativa. Trataba de iniciar de forma simultánea el tratamiento curativo y paliativo (no siendo un modelo excluyente), de forma que junto al tratamiento necesario

Figura 1. Modelos de cuidados paliativos ${ }^{9}$

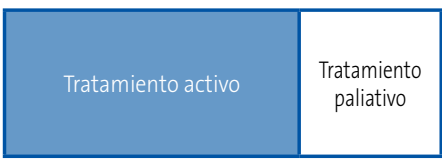

Modelo de cuidados paliativos según la Organización Mundial de la Salud (1990)

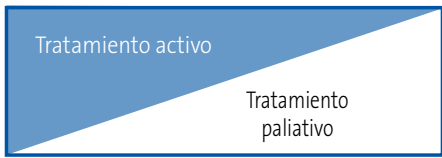

Modelo de cuidados paliativos según la el Children's Hospice International (1993)

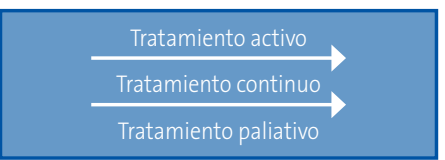

Modelo de cuidados paliativos según la Organización Mundial de la Salud (1998) 
para su enfermedad de base se asociaban tratamientos de soporte que permitían al paciente una mejor tolerancia al tratamiento y una mejoría en la calidad de vida (Fig. 1), evitando hacer una distinción rígida entre los distintos tratamientos curativos y paliativos. A su vez, en ese momento se incluyó, dentro de los CPP, el seguimiento de la familia, tratando de prolongarlo hasta terminar el duelo.

En 1998 la OMS insistió en que el objetivo principal de los CPP era lograr la mejor calidad de vida del niño y de su familia, tratando de forma integral al paciente, no solo controlar el dolor sino también el resto de síntomas acompañantes ocasionados por la enfermedad y derivados de la misma como los problemas psicológicos, sociales y espirituales. Recomendaba administrar de forma conjunta y simultánea al tratamiento curativo las terapias complementarias de apoyo, debiendo comenzar estas últimas cuando se diagnosticaba la gravedad de la enfermedad y continuar con ellas de forma independiente a si el niño recibe o no tratamiento dirigido a la curación (Fig. 1). En el año 2000, la Academia Americana de Pediatría propuso de forma similar un modelo donde se iniciaran de forma conjunta los CPP con el tratamiento curativo, independientemente de si el objetivo era la curación o no9 .

\section{PACIENTES SUSCEPTIBLES DE CUIDADOS PALIATIVOS PEDIÁTRICOS}

Previo a la instauración de los últimos modelos descritos en el apartado anterior, el paciente que se incluía en los programas de CPP se encontraba en situación terminal, definida como el que presentaba una enfermedad avanzada e incurable (pronóstico de vida inferior a seis meses), con múltiples síntomas derivados de su enfermedad y gran impacto emocional tanto en el paciente como en la familia.

Sin embargo, esta definición clásica deja fuera a un amplio número de pacientes. En la actualidad, no se considera de forma tan estanca el "factor pronóstico vital" (pronóstico de vida inferior a seis meses) para que un paciente sea aceptado en una unidad de CPP, aunque si tendrá influencia en las diversas actuaciones a realizar durante su seguimiento.

La Asociación de Niños con Situación que Amenaza la Vida o Situación de Terminalidad y sus Familias (ACT) (Children with Life-Threatening or Terminal Conditions and their Families) ha desarrollado una guía para el desarrollo de los Servicios de Cuidados Paliativos Pediátricos que clasifica a los niños en cuatro categorías para recibir tratamiento paliativo (Tabla 1$)^{10}$

Ello no significa que la totalidad de los pacientes, a lo largo de su evolución clínica, deben ser atendidos por servicios específicos de CPP. Se pueden diferenciar tres niveles de atención (Tabla 2$)^{11}$.

\section{PROPORCIONAR CUIDADOS PALIATIVOS PEDIÁTRICOS}

La Asociación Europea de Cuidados Paliativos publicó, en 2007, unos estándares de cuidados paliativos pediátricos donde se aborda la asistencia integral al niño y su familia como unidad. Finalmente afirman que es esencial que se aplique el texto en toda Europa ${ }^{12}$.

Sin embargo, el Consejo de Europa sobre cuidados paliativos emitió un documento donde reconocía que los CPP especializados no se ofrecían en la amplia mayoría de los países europeos, aunque destacaba que existían importantes iniciativas en este aspecto $^{13}$

Se postuló un modelo de cuidados paliativos del Sistema Nacional de Salud, en el año 2007, integrado en la red asistencial de sistema sanitario. El modelo identificaba los niños como población diana.

En España existen tres unidades de CPP reconocidas como tales: la primera, creada en 1991, en el Hospital Sant Joan de Déu de Barcelona, la segunda, en 1997 en el Hospital Universitario Materno Infantil de Canarias y, en el 2008, se abrió la Unidad del Hospital Niño Jesús de Madrid ${ }^{14}$. También cabe destacar que la Unidad de Oncología Pediátrica del Hospital La Fe, desde 1997, administra, de forma pionera en nuestro país, cuidados paliativos 


\begin{tabular}{|l|l|}
\hline \multicolumn{2}{|l|}{ Tabla 1. Categorias para recibir cuidados paliativos pediátricos } \\
\hline Grupos & Definición \\
\hline Grupo 1 & $\begin{array}{l}\text { Se trata de niños que presentan enfermedades graves, pero en las que el tratamiento curativo es posible, } \\
\text { aunque puede fracasar (por ejemplo, cáncer infantil) }\end{array}$ \\
\hline Grupo 2 & $\begin{array}{l}\text { Enfermedades donde la muerte prematura es ineludible, pero se realizan largos periodos de tratamiento } \\
\text { intensivo destinado a aumentar el periodo de vida y el paciente tiene la posibilidad de realizar actividades } \\
\text { cotidianas (por ejemplo, fibrosis quística, infección por virus de la inmunodeficiencia humana, enfermedad } \\
\text { de Duchenne) }\end{array}$ \\
\hline Grupo 3 & $\begin{array}{l}\text { Enfermedades progresivas, que no tienen opciones de tratamiento curativo, donde el tratamiento es } \\
\text { exclusivamente paliativo y puede prolongarse largo tiempo (por ejemplo, atrofia muscular espinal) }\end{array}$ \\
\hline Grupo 4 & $\begin{array}{l}\text { Enfermedades en situación irreversible pero no progresiva, con complejas necesidades sanitarias, que causan } \\
\text { gran discapacidad y producen complicaciones de salud e incrementan la probabilidad de muerte prematura } \\
\text { (por ejemplo, parálisis cerebral, síndromes polimalformativos) }\end{array}$ \\
\hline
\end{tabular}

Tabla 2. Niveles de atención en cuidados paliativos pediátricos

\begin{tabular}{|l|l|l|}
\hline \multicolumn{1}{|c|}{ Nivel } & \multicolumn{1}{|c|}{ Tipo de cuidado paliativo } & \multicolumn{1}{c|}{ Definición } \\
\hline Primer nivel & Enfoque de los cuidados paliativos & $\begin{array}{l}\text { Cualquier profesional sanitario puede facilitar el enfoque paliativo } \\
\text { de la enfermedad }\end{array}$ \\
\hline Segundo nivel & Cuidados paliativos generales & $\begin{array}{l}\text { Saber manejar los problemas más comunes; deben ser } \\
\text { administrados por algunos profesionales que han recibido } \\
\text { formación específica a este nivel (se comparten los cuidados con la } \\
\text { Unidad de Cuidados Paliativos Pediátricos) }\end{array}$ \\
\hline Tercer nivel & Cuidados paliativos especiales & $\begin{array}{l}\text { Existe una gran complejidad a nivel clínico y también a nivel } \\
\text { personal, familiar o social, que hacen precisa la actuación de } \\
\text { servicios especializados en cuidados paliativos pediátricos }\end{array}$ \\
\hline
\end{tabular}

para niños con cáncer en domicilio, no solo atendiendo en la fase final, si no apoyando en las diversas etapas del tratamiento y permitiendo una disminución de los días de hospitalización ${ }^{15,16}$.

A su vez, existen diversas iniciativas que proporcionan CPP con diversos mecanismos organizativos, generalmente asociadas a los Servicios de Oncología Infantil, como es el caso del Hospital Virgen del Rocío en Sevilla y el Hospital Vall d'Hebron en Barcelona ${ }^{14}$.

\section{ALIVIO DEL SUFRIMIENTO FÍSICO. APORTACIONES DE LOS CPP}

El control de síntomas es una de las partes más importantes y el punto que quizás más atención demanda de los cuidados paliativos. El tratamiento del cáncer y de otras enfermedades amenazantes para la vida ha presentado en los últimos años importantes mejoras. Sin embargo, un porcentaje de ellos fallecen y presentan diversas complicaciones en la evolución de su enfermedad. Es importante la formación en este ámbito para saber reconocer los signos que se presentan y tratarlos de la forma más adecuada posible.

Pocos trabajos abordan los problemas de los niños al final de la vida. Por el contrario, sí existen estudios sobre el manejo de los síntomas en los adultos, en la mayoría de ocasiones subóptimo.

El estudio realizado por Joanne Wolfe et al. en el Children's Hospital de Boston en 1998 fue uno de los primeros estudios realizados en CPP. Realizaron un análisis multivariante donde observaron que la baja implicación de los oncólogos pediátricos en el tratamiento del dolor en los últimos días de vida de los pacientes suponía un factor de riesgo independiente para que estos sufrieran más dolor ${ }^{17}$.

Sin embargo, no solo el dolor, también se incrementa el sufrimiento en el aspecto físico, psicológico, social y emocional cuando se someten a los diferentes tratamientos. Desafortunadamente, menos del 30\% refieren que el control del dolor y el alivio de síntomas sean adecuados ${ }^{17}$. Cabe destacar que, aparentemente, esa tendencia está cambiando. En 
los últimos años se observa una mayor implicación no solo a nivel clínico sino también psicológico, aunque en España no conocemos datos concretos al respecto.

Para un abordaje completo se debe realizar ${ }^{9}$ :

- Una correcta evaluación etiológica que nos permita establecer la causa del problema y en consecuencia un adecuado manejo del mismo.

- En el momento de instaurar un tratamiento, valorar la relación daño/beneficio según la fase evolutiva del paciente y la repercusión que suscita sobre su calidad de vida.

- Evitar el exceso de medicación, eliminando aquellos fármacos que no sean necesarios.

- Elegir terapias de fácil cumplimentación, tratando de mantener la vía oral siempre que sea posible para el paciente.

El dolor es el síntoma más frecuente y el que más repercusión suele tener tanto en el paciente como en su familia. Un diagnóstico tardío del dolor, la subestimación del mismo o el miedo a la adicción a opioides son factores que juegan un papel importante para el mal control del mismo. Aunque no deben descuidarse el resto, como la disnea, los vómitos, las náuseas...

No existen escalas universales para medirlos. Se han desarrollado diversos instrumentos para medir el sufrimiento, el dolor, la ansiedad o la fatiga. Sin embargo, la mayoría de ellos han sido validados en otro tipo de población. Se pueden utilizar para obtener una evaluación objetiva y más precisa de los síntomas y de la respuesta al tratamiento.

\section{LA FAMILIA}

A lo largo de los años este concepto ha ido evolucionando en función de los cambios sociales y culturales, modificando la estructura familiar clásica. Por tanto, se ha de partir de un amplio concepto de la misma, que pueda incluir los distintos modelos estructurales.

El diagnóstico de una enfermedad terminal en un niño produce un profundo impacto en las familias y la forma en la que esta se adapta y responde a la enfermedad influye de manera clara en la calidad de vida del enfermo ${ }^{18}$. En esta situación, se plantean dos cuestiones principales: tratar de satisfacer las necesidades emocionales y físicas del enfermo y mantener el normal funcionamiento de la familia.

Si un niño padece una enfermedad, sobre todo si es grave, la vida familiar se modifica y se estructura alrededor de los cuidados que precisa el niño.

En muchas familias los hermanos pasan a un segundo plano; es importante acordarse de ellos, muchas veces son los grandes olvidados. Su atención debe comenzar con el diagnóstico y debe ser una parte fundamental de los CPP.

Se han publicado diversos estudios donde se observa que las pérdidas súbitas e inesperadas aumentan la morbilidad psicológica a largo plazo en los familiares, a diferencia de los que tienen mayor periodo adaptativo, siendo en el último caso más marcada la presencia de depresión en los padres que en las madres ${ }^{19}$.

Si la muerte del niño es el final natural de una enfermedad ya conocida, en muchas ocasiones tanto el paciente como la familia han contemplado este final como posibilidad, lo cual proporciona tiempo para prepararse. La anticipación del duelo es un problema de ajuste psicológico por el que en ocasiones pasan los familiares, pero no supone en absoluto beneficio para el paciente.

Un ejemplo de patología donde la familia presenta mayor periodo adaptativo es la enfermedad oncológica, en estas familias se puede introducir el modelo family adjustment and adaptation response (FAAR). En este modelo, desarrollado por Patterson en 1988, con base en familias con situaciones estresantes, el paciente y la familia consiguen un balance entre los factores estresantes, las capacidades familiares y las conductas que deben seguir ${ }^{18}$. Normalmente cada miembro de la familia y el propio paciente tienen sus factores o situaciones desencadenantes del desajuste (Tabla 3). Sin embargo, el paciente y los padres son los que más manifiestan el desajuste ${ }^{18}$. 
Tabla 3. Factores desencadenantes del desajuste familiar

- El momento del diagnóstico y el tratamiento durante los cuales suelen experimentar temor, pérdida de control y necesidad de ayuda

- Disminución o pérdida de independencia de sus hijos

- Sentimientos de culpa por no haber sabido proteger a sus hijos

- Forma de comunicarse con el niño sobre el diagnóstico y el tratamiento de la enfermedad

- Dificultades para que sus hijos tomen de forma adecuada la medicación

- Disminución o falta de apoyo del resto de la familia

\section{SEDACIÓN TERMINAL}

En el desarrollo de los distintos procesos patológicos, puede producirse una inflexión en la evolución del paciente, manifestada como una aceleración de la enfermedad y un empeoramiento clínico con ausencia de respuesta a los sucesivos tratamien$\operatorname{tos}^{19-22}$

A su vez, en la fase final pueden aparecer síntomas difíciles de controlar pese a la intensificación de las medidas terapéuticas, incluso síntomas refractarios que nos indicarían la necesidad de iniciar una sedación paliativa con la finalidad de mejorar el confort del paciente.

En estas situaciones se debe comunicar en la medida de lo posible al niño o adolescente del procedimiento que se va a realizar (si su edad, desarrollo y madurez lo permiten) y obtener el consentimiento informado del tutor o tutores legales ${ }^{21}$.

\section{DISCUSIÓN}

Como hemos comentado previamente, los CPP pretenden una atención integral del niño y su familia teniendo en cuenta los aspectos emocionales, físicos, sociales y espirituales.
Son beneficiosos cuando el tratamiento de la enfermedad no consigue la curación del paciente, logrando una mejor calidad de vida del niño y su familia.

No deben basarse únicamente en los pacientes oncológicos, puesto que como hemos podido comprobar a lo largo del trabajo, en los últimos años se ha observado un aumento en la prevalencia de pacientes con enfermedades complejas y evolución limitada.

Finalmente, la Academia Americana de Pediatría resume los CPP quizás de la manera mejor y más simple: "Cuidados Paliativos es agregar vida a los años del niño, y no solo años a su vida".

\section{CONFLICTO DE INTERESES}

Los autores declaran no presentar conflictos de intereses en relación con la preparación y publicación de este artículo.

\section{ABREVIATURAS}

ACT: Asociación de Niños con Situación que Amenaza la Vida o Situación de Terminalidad y sus Familias - CCP: cuidados paliativos pediátricos $\bullet \mathrm{CP}$ : cuidados paliativos $\bullet$ OMS: Organización Mundial de la Salud. 


\section{BIBLIOGRAFÍA}

1. Goldman $A . A B C$ of palliative care. Specials problems of children. BMJ. 1998;316:49-52.

2. Monleón Luque M, Rus Palacios M, Martino Alba R. Cuidados paliativos pediátricos: una necesidad y un reto. Bol Pediatr. 2010;50:108-12.

3. Beauchamp TL, Childress JF. Principles of biomedical ethics. New York: Oxford University Press; 2001.

4. Martín Rodrigo JM, Gabaldón Fraile S, TriasJover M, Gascón Romero J. Paciente pediátrico y cuidados paliativos. En: Sociedad Española de Cuidados Paliativos [en línea] [consultado el 09/09/2015]. Disponible en www.secpal.com/PACIENTE-PEDIA TRICO-Y-CUIDADOS-PALIATIVOS

5. Wolfe J, Friebert S, Hilden J. Caring for children with advanced cancer integrating palliative care. Pediatr Clin North Am. 2002;49:1043-62.

6. Martino Alba R. ¿Qué debemos saber sobre los cuidados paliativos pediátricos en los niños? En: AEPap. Curso de Actualización en Pediatría 2012. Madrid: Exlibris Ediciones; 2012. p. 285-92.

7. Davies B, Sehring SA, Colin Partridge J, Cooper BA, Hughes A, Philp JC, et al. Barriers to palliative care for children: perceptions of pediatric health care providers. Pediatrics. 2008;121;282-8.

8. Organización Mundial de la Salud. Alivio del dolor y tratamiento paliativo en cáncer. Informe de un Comité de Expertos. Ginebra: OMS; 1990.

9. Villegas Rubio JA, Antuña García MJ. Cuidados paliativos pediátricos. Bol Pediatr. 2012;52:131-45.

10. Association for Children with Life-Threatening or Terminal Conditions and their Families (ACT), Royal College of Paediatrics and Child Health. A guide to the development of children's palliative care services. London: Royal College of Paediatrics and Child Health; 2003.

11. Martino Alba R. El proceso de morir en el niño y el adolescente. Pediatr Integral. 2007;11:926-34.

12. Craig F, Abu-Saad Huijer H, Benini F, Kuttner L, Wood C, Feraris PC, et al. IMPaCCT: standards for paediatric palliative care in Europe. Schmerz. 2008;22:401-8.
13. European Parliament's Committee on the Environment, Public Health and Food Safety. The external report. Palliative Care in the European Union. Houston: IAHPC Press; 2007.

14. Martino R, Payeras J, Rodríguez S, Rus M. Estado actual de los cuidados paliativos en España. Med Pal. 2009;16;298-302.

15. Fernández Navarro JM, Barahona Clemente $A$, Cañete Nieto A, Ortí Martínez P, Pozuelo Muñoz B, Castel Sánchez V. Hospitalización domiciliaria para niños con cáncer: un paso más hacia la atención integral en oncología pediátrica. An Esp Pediatr. 1998;48:1-3.

16. Fernández Navarro JM, Pozuelo Muñoz B, Ortí Martínez P, López Ferrer L, Cañete Nieto A, Verdeguer Miralles $V$, et al. Evaluación de un programa de hospitalización a domicilio para niños con cáncer. An Esp Pediatr. 2000;52:41-6.

17. Wolfe J, Grier HE, Klar N, Levin SB, Ellenbogen JM, Salem-Schatz $S$, et al. Symptoms and suffering at the end of life in children with cancer. N Engl J Med. 2000;342:326-33.

18. Patterson J, Holm K, Gurney Y. The impact of childhood cancer on the family: A qualitative analysis of strains, resources, and coping behaviors. Psychooncology. 2004;13:390-407.

19. Valdimarsdóttir $U$, Kreicbergs $U$, Hauksdóttir A, Hunt $\mathrm{H}$, Onelöv E, Henter JI, et al. Parents' intellectual and emotional awareness of their child's impending death to cancer: a population-based long-term follow-up study. Lancet Oncol. 2007;8:706-14.

20. Vrakking AM, van der Heide A, Arts WF, Pieters R, van der Voort E, Rietjens JA, et al. Medical end-of-life decisions for children in the Netherlands. Arch Pediatr Adolesc Med. 2005;159:802-9.

21. Hinds PS, Drew D, Oakes LL, Fouladi M, Spunt SL, Church C, et al. End-of-life care preferences of pediatric patients with cancer. J Clin Oncol. 2005; 23:9146-54.

22. Duarte IC. El niño con patología oncológica en la etapa final de su vida. Rev Gastrohnup. 2012;14: S19-S24. 